\title{
CAPACIDADE DE INFERÊNCIA DIAGNÓSTICA DE ENFERMEIROS ESPECIALISTAS $^{1}$
}

\section{DIAGNOSTIC INFERENCE CAPACITY OF SPECIALIST NURSES}

\section{CAPACIDAD DE INFERENCIA DIAGNÓSTICA DE ENFERMERAS ESPECIALISTAS}

\author{
Maria Isabel da Conceição Dias Fernandes* \\ Aryele Rayana Antunes de Araújo ** \\ Cecília Maria Farias de Queiroz Frazão ${ }^{* * *}$ \\ FERnANDA BeATriz Batista Lima E SiLVA ${ }^{* * *}$ \\ Bertha Cruz Enders ${ }^{* * * * *}$ \\ Ana Luisa Brandẽo de CARVAlHo LiRA ${ }^{* * * * *}$
}

\begin{abstract}
RESUMO
Objetivo: Verificar a capacidade de inferência diagnóstica de enfermeiros especialistas. Método: Estudo descritivo, realizado em julho de 2013, com cinco enfermeiros especialistas do grupo de pesquisa intitulado Práticas Assistenciais e Epidemiológicas em Saúde e Enfermagem. A capacidade de inferência diagnóstica foi avaliada por meio dos testes de eficiência, taxa de falso positivo, taxa de falso negativo e tendência. A pesquisa foi aprovada pelo comitê de ética em pesquisa da instituição responsável, por meio do protocolo No 148.428. Resultados: Dentre os especialistas avaliados quanto a sua capacidade de inferência diagnóstica, apenas dois atingiram as pontuações desejáveis dos quatro atributos avaliados. Conclusão: Conclui-se que poucos enfermeiros especialistas obtiveram resultado satisfatório. Assim, é necessário o desenvolvimento de estratégias de aprendizado desde a graduação e a realização de educação continuada para os profissionais da prática.
\end{abstract}

Palavras chave: Enfermagem, diagnóstico de enfermagem, avaliação em enfermagem, estudos de avaliação.

\footnotetext{
ABSTRACT

Objective: To assess the capacity for diagnostic inference among specialist nurses. Method: Descriptive study, performed in July 2013, with five specialist nurses of the research group entitled Care and Epidemiology Prac-

${ }^{1}$ Artigo extraído da dissertação "Acurácia dos indicadores clínicos do diagnóstico de enfermagem volume de líquidos excessivo em pacientes submetidos à hemodiálise”, 2014, Universidade Federal do Rio Grande do Norte, Brasil.

* Enfermeira, Mestre, Doutoranda, Programa de Pós-Graduação em Enfermagem, Universidade Federal do Rio Grande do Norte/PPGENF/UFRN. Natal (RN), Brasil. Email: bebel_6@hotmail.com. Autor de correspondência.

${ }^{* *}$ Enfermeira, Mestre, Universidade Federal do Rio Grande do Norte/PPGENF/UFRN. Natal (RN), Brasil. Email: aryelearaujo_ufrn@yahoo.com.br

${ }^{* * *}$ Enfermeira, Doutora, Professora Auxiliar do Departamento de Enfermagem, Universidade Federal de Pernambuco/ UFPE. Recife (PE), Brasil. Email: ceciliamfqueiroz@gmail.com

${ }^{* * * *}$ Enfermeira, Mestre, Doutoranda, Programa de Pós-Graduação em Enfermagem, Universidade Federal do Rio Grande do Norte/PPGENF/UFRN. Natal (RN), Brasil. Email: fbeatrizlima@hotmail.com

${ }_{* * * * *}$ Enfermeira, Professora Doutora, Departamento de Enfermagem, Universidade Federal do Rio Grande do Norte/UFRN. Natal (RN), Brasil. Email: berthacruz.enders@gmail.com

${ }_{* * * * *}$ Enfermeira, Professora Doutora, Departamento de Enfermagem, Universidade Federal do Rio Grande do Norte/UFRN. Natal (RN), Brasil. Email: analuisa_brandao@yahoo.com.br
} 
tices in Health and Nursing. The diagnostic inference capacity was assessed through efficiency tests, false positive rate, false negative rate and trend analysis. The research was approved by the research ethics committee of the sponsoring institution, through the Protocol $N^{\circ} 148.428$. Results: Among the specialists evaluated for their diagnostic inference capacity, only two reached the desirable scores out of the four attributes evaluated. Conclusion: It is concluded that few specialist nurses obtained satisfactory results. Thus, it is necessary to develop learning strategies in their undergraduate studies and their continuing education for practitioners.

Key words: Nursing, nursing diagnosis, nursing assessment, evaluation studies.

\section{RESUMEN}

Objetivo: Comprobar la capacidad de inferencia diagnóstica de enfermeras especialistas. Método: Estudio descriptivo, llevado a cabo en julio de 2013, con cinco enfermeras especialistas del grupo de investigación titulado Prácticas de Atención y Epidemiológicas en Salud y Enfermería. La capacidad de inferencia diagnóstica se evaluó a través de pruebas de eficiencia, tasa de falsos positivos, falsos negativos y tendencia. La investigación fue aprobada por el comité de ética en investigación de la institución responsable, a través del Protocolo $\mathrm{N}^{\circ} 148.428$. Resultados: Entre los especialistas evaluados como su capacidad de inferencia diagnóstica, sólo dos alcanzaron los puntajes deseables de los cuatro atributos. Conclusión: Se concluye que pocas enfermeras especialistas obtuvieron resultados satisfactorios. Por lo tanto, es necesario desarrollar estrategias de aprendizaje desde su graduación y la educación continua de los profesionales en la práctica.

Palabras clave: Enfermería, diagnóstico de enfermería, evaluación en enfermería, estudios de evaluación.

Fecha recepción: 23/04/15 Fecha aceptación: 16/12/16

\section{INTRODUÇÃO}

A utilização de diagnósticos de enfermagem pelos enfermeiros possibilita que as respostas humanas às condições de saúde/doença dos indivíduos/comunidade sejam julgadas e estabelecidas intervenções de enfermagem, com vistas a atingir resultados positivos de saúde (1). Assim, ao empregar diagnósticos de enfermagem no cuidado ao paciente, as ações referentes às intervenções e resultados traçados podem ser visualizadas com mais clareza e a comunicação entre os profissionais da saúde é facilitada (2).

Para o agrupamento desses diagnósticos e representação desse conhecimento na ciência, de modo a estruturar uma linguagem padronizada de comunicação, são utilizadas as taxonomias. Nesse sentido, a terminologia da NANDA Internacional como taxonomia dos diagnósticos de enfermagem possibilita uma linguagem compartilhada entre os enfermeiros sobre os problemas de saúde, estado de risco e disposição para a promoção da saúde dos indivíduos. Essa taxonomia é utilizada globalmente e traduzida em 16 idiomas. Seus diagnósticos são compostos por enunciado diagnóstico; definição do enunciado; fatores relacionados/risco; e características definidoras (1).

Estudo revela que as taxonomias fornecem suporte ao raciocínio clínico do enfermeiro por ser uma estrutura formal e organizada do conhecimento de enfermagem (3). Nesse aspecto, é o raciocínio clínico que permite ao enfermeiro a seleção do diagnóstico de enfermagem que melhor se coaduna à resposta de saúde/doença apresentada pelos indivíduos, conduzindo ao processo de acurácia diagnóstica (4).

A acurácia diagnóstica reflete a validade e a confiabilidade dos dados coletados e interpretados pelo enfermeiro. A identificação de 
diagnósticos de enfermagem acurados pelo enfermeiro é uma ferramenta importante para a escolha de intervenções adequadas ao problema apresentado pelo paciente, de modo que resultados positivos de saúde sejam alcançados com eficiência (5). Em contraponto, a imprecisão diagnóstica repercute em todas as etapas do cuidar no âmbito da enfermagem, pois podem ser traçadas intervenções impróprias a partir de diagnósticos traçados imprecisamente, pondo em risco a qualidade do cuidado.

Nesse aspecto, a identificação diagnóstica na enfermagem ainda possui grandes fragilidades se confrontada com a medicina (6). $\mathrm{Na}$ enfermagem não existem padrões de referência com alto nível de acurácia como na medicina que utiliza dispositivos que permitem uma inferência diagnóstica altamente precisa. Como os diagnósticos de enfermagem são traçados a partir da observação/mensuração de respostas humanas, as quais não são verificadas a partir de aparelhos que garantem com precisão a sua presença ou ausência, a identificação de diagnósticos na enfermagem dependerá quase exclusivamente da capacidade diagnóstica dos enfermeiros. Para tanto, a capacitação do enfermeiro é primordial para essa atividade (7).

Destarte, para realizar uma inferência diagnóstica correta, o enfermeiro deverá identificar se as características definidoras e fatores relacionados/risco são condizentes com os dados apresentados pelo paciente investigado. Dessa maneira, deve estabelecer bons indicadores clínicos para a identificação de diagnósticos de enfermagem com maior acurácia (2). Para que esse processo seja fidedigno é necessário que o enfermeiro tenha conhecimento acerca dos diagnósticos de enfermagem e possua competências nos domínios intelectual, interpessoal e técnico, além de habilidades mentais e cognitivas (8).

Entretanto, apesar das implicações inerentes, a acurácia diagnóstica não é uma das principais preocupações dos enfermeiros, bem como não está sendo abordada na educação, sendo importante o desenvolvimento de estudos envolvendo a precisão diagnóstica (8).

Nessa perspectiva, compreende-se como importante reconhecer aqueles enfermeiros com boa capacidade de inferência diagnóstica, os quais estarão mais aptos a realizar o julgamento diagnóstico. Assim, a verificação da eficiência, a taxa de falso positivo, a taxa de falso negativo e a tendência, são atributos capazes de avaliar o desempenho dos enfermeiros especialistas, garantindo uma acurácia diagnóstica com menos vieses (7).

Nesse sentido, este estudo questiona: existe acurácia por parte de enfermeiros na determinação dos diagnósticos de enfermagem da NANDA Internacional? Dessa forma, traçou-se como objetivo verificar a capacidade de inferência diagnóstica de enfermeiros especialistas.

\section{MÉTODO}

Estudo descritivo, realizado com cinco enfermeiros especialistas do grupo de pesquisa intitulado Práticas Assistenciais e Epidemiológicas em Saúde e Enfermagem (PAESE), o qual desenvolve atividades sobre Sistematização da Assistência de Enfermagem (SAE), vigilância epidemiológica no âmbito hospitalar e na atenção primária à saúde.

Os enfermeiros do grupo foram avaliados a partir do currículo Lattes, sendo selecionados para a participação no estudo aqueles com pesquisas publicadas sobre diagnósticos de enfermagem e/ou prática clínica e/ou de ensino sobre nefrologia, tendo-se em vista que esses enfermeiros julgariam a presença do Volume de líquidos excessivo em casos clínicos contemplando pacientes renais crônicos.

Os especialistas selecionados receberam uma carta convite por meio do correio eletrônico, contendo esclarecimentos sobre os objetivos da pesquisa, disponibilidade em participar de um treinamento e em responder o instrumento proposto. Posteriormente 
o aceite em participar do estudo, o Termo de Consentimento Livre e Esclarecido (TCLE) foi enviado para cada participante, termo que garante o sigilo das informações colhidas e a anuência do participante.

Anteriormente a verificação da inferência diagnóstica, realizou-se um treinamento com carga horária de cinco horas, ministrado pela pesquisadora, sobre acurácia diagnóstica, raciocínio clínico, inferência diagnóstica, pacientes com insuficiência renal crônica e o diagnóstico de enfermagem Volume de líquidos excessivo, com vistas a minimizar o viés da inferência diagnóstica.

Apesar dos enfermeiros serem especialistas, existe a necessidade de treinamento devido ao fato da enfermagem não possuir padrões de referência perfeitos e não conter instrumentos que garantam com fidedignidade a presença ou a ausência das respostas humanas (7).

Para verificar a inferência diagnóstica dos especialistas utilizou-se o método de classificação de atributos que verifica a capacidade de um indivíduo classificar corretamente dois estados (9). Para avaliar o desempenho de cada especialista consideram-se quatro atributos, quais sejam: a eficiência, a taxa de falso positivo, a taxa de falso negativo e a tendência (9).

Aplicando-se aos diagnósticos de enfermagem, a taxa de falso negativo (FN) é a probabilidade de um indivíduo com o diagnóstico de enfermagem ser identificado como não o tendo, cuja fórmula é $\mathrm{FN}=\mathrm{No}$ de indivíduos com o diagnóstico de enfermagem incorretamente classificado/No total de indivíduos com o diagnóstico de enfermagem. A taxa de falso positivo (FP) é a probabilidade de se identificar um diagnóstico de enfermagem em um indivíduo que não o possui, com fórmula: $\mathrm{FP}=\mathrm{No}$ de indivíduos sem o diagnóstico de enfermagem erroneamente classificado como tendo/No total de indivíduos sem o diagnóstico de enfermagem $(7,9)$.

A eficiência (E) refere-se à capacidade de um diagnosticador detectar corretamen- te a presença ou ausência do diagnóstico de enfermagem, sendo calculada pela fórmula: $\mathrm{E}=\mathrm{N}^{\circ}$ de identificações corretas/No total de casos. E, por fim, a tendência (T) representa o viés de o diagnosticador classificar um indivíduo como tendo ou não o diagnóstico $(\mathrm{T}=\mathrm{T}[\mathrm{FP}] / \mathrm{T}[\mathrm{FN}])(7)$.

Para essa avaliação são fornecidas histórias verdadeiras e falsas para cada especialista, os quais assinalam a presença ou ausência do diagnóstico de enfermagem de acordo com seu julgamento (7). O número de histórias distribuídas depende da quantidade de participantes. Dessa forma, quando o número de diagnosticadores for igual ou maior que três, devem ser confeccionados 12 casos, os quais deverão ser repetidos três vezes, aleatoriamente, de forma que cada diagnosticador realize a inferência diagnóstica em 36 casos (9).

Desse modo, como a amostra deste estudo foi composta por cinco diagnosticadores, 12 histórias foram distribuídas. Essas histórias clínicas eram fictícias, criadas pela pesquisadora do estudo, e continham as características definidoras do diagnóstico de enfermagem Volume de líquidos excessivo em pacientes com doença renal crônica. Ressalta-se que na metade das histórias o diagnóstico supracitado estava presente e na outra metade ausente. Cada especialista ao realizar a leitura de cada história clínica deveria julgar a presença ou ausência do Volume de líquidos excessivo de acordo com as características definidoras presentes nos pacientes dos casos e assinalar sua decisão.

As 12 histórias clínicas foram fornecidas e analisadas três vezes por cada especialista, sendo distribuídas de modo aleatório em cada rodada (7). Ressalta-se que em cada rodada, as folhas contendo as histórias clínicas, eram recolhidas dos participantes para impedir que esses fossem influenciados pelas respostas anteriores. Além das histórias clínicas, os especialistas responderam um questionário com perguntas relacionadas aos dados sociodemográficos e da atuação profissional, com vistas à caracterização da amostra. 
As respostas fornecidas por cada especialista nas três rodadas foram tabuladas no $\mathrm{Mi}$ crosoft Excel 2010 e posteriormente comparadas conforme o gabarito da pesquisadora, sendo calculadas as medidas dos atributos a partir das respostas dos participantes. Os resultados obtidos foram comparados aos parâmetros expostos na Tabela 1 a seguir.

Tabela 1. Parâmetros para a avaliação da capacidade de inferência diagnósticade enfermeiros especialistas. Natal, Rio Grande do Norte/Brasil, 2013.

\begin{tabular}{lccc}
\hline Parâmetros & Aceitável & Marginal & Inaceitável \\
\hline Eficácia & 0,9 ou mais & $>0,8-0,9$ & Menos de 0,8 \\
Falso Positivo & 0,05 ou menos & $\leq 0,10$ & Mais que 0,10 \\
Falso Negativo & 0,02 ou menos & $\leq 0,10$ & Mais que 0,10 \\
Tendência & $0,80-1,20$ & $0,50-0,80$ ou $1,2-1,5$ & Menos que 0,50 ou mais que 1,5 \\
\hline
\end{tabular}

Fuente: Hradesky, 1989 (9).

Classificou-se o resultado de cada especialista em: marginal, inaceitável ou aceitável (8). Neste estudo, para que os enfermeiros fossem classificados como aptos utilizou-se como ponto de corte a classificação marginal.

A pesquisa foi aprovada pelo comitê de ética em pesquisa da instituição responsável, por meio do protocolo No 148.428, sob o Certificado de Apresentação para Apreciação Ética (CAAE) No 08696212.7.0000.5537.

\section{RESULTADOS}

Relativo à caracterização da amostra 80\% eram do sexo feminino, a idade variou entre 23 e 39 anos, a experiência profissional variou entre três a doze anos, 40\% trabalhavam na assistência, 40\% eram bolsistas de pósgraduação e 20\% eram docentes. No que tange à titulação, 60\% eram mestres e 40\% especialistas e mestrandos.

Em relação à capacidade para traçar diagnósticos de enfermagem, 60\% considerou essa habilidade de 50 a 75\% acuradas. No entanto, ao se avaliar a capacidade de inferência diagnóstica de cada especialista, apenas dois (especialistas 2 e 3 ) apresentaram uma boa capacidade para inferir diagnósticos de enfermagem, conforme demonstra a Tabela 2 abaixo.

Tabela 2. Resultado da avaliação da capacidade de inferência diagnóstica realizada após o treinamento com os diagnosticadores. Natal, Rio Grande do Norte/Brasil, 2013 ( $\mathrm{n}=5$ ).

\begin{tabular}{lcccc}
\hline Volume de líquidos excessivo & FP & FN & Eficácia & Tendência \\
\hline Diagnosticador 1 & 0 & 0.166 & 0.916 & 0 \\
Diagnosticador 2 & 0 & 0 & 1 & 1 \\
Diagnosticador 3 & 0 & 0.055 & 0.972 & 1 \\
Diagnosticador 4 & 0 & 0.166 & 0.916 & 0 \\
Diagnosticador 5 & 0.166 & 0 & 0.916 & - \\
\hline
\end{tabular}

Legenda: FN: Taxa de falso negativo; FP: Taxa de falso positivo. 
Os demais especialistas apresentaram valores inaceitáveis nas taxas de tendência e nas taxas de falso positivo e falso negativo.

\section{DISCUSSÃO E CONCLUSÃO}

Referente à avaliação da capacidade de inferência diagnóstica dos especialistas deste estudo, dentre os cinco investigados, apenas dois atingiram os pontos de corte, embora, a maioria tenha afirmado que sua habilidade diagnóstica era de 50 a $75 \%$ acuradas. Os erros principais cometidos pelos especialistas estiveram relacionados às taxas de falso positivo e falso negativo, dado que indica a incerteza dos participantes ao analisar as características definidoras presentes em cada história clínica e considerá-las como indicadores para o diagnóstico elencado nos casos.

Estudo similar com seis enfermeiros especialistas identificou que apenas dois foram considerados aptos ao serem avaliados em relação aos atributos da inferência diagnósti$\mathrm{ca}$, apesar de terem recebido também treinamento sobre diagnósticos de enfermagem e/ ou terem utilizado os diagnósticos na prática clínica/pesquisa. Como justificativa para esse achado, os autores destacam que esse resultado pode estar atrelado à subjetividade das características definidoras (2).

Ratificando os achados deste estudo, pesquisa com enfermeiros docentes e assistencialistas verificou dados similares. Ao serem indagados sobre sua capacidade de inferência diagnóstica, os enfermeiros autoavaliaram essa habilidade como $70 \%$ acurada, entretanto, quando essa capacidade foi medida verificou-se uma baixa precisão diagnóstica (10). Nesse sentido, pesquisa afirma que os enfermeiros necessitam desenvolver com melhor desenvoltura suas habilidades para a identificação diagnóstica acurada, com vistas a elencar intervenções eficazes (5).

Essa realidade pode ser um reflexo do ensino dos diagnósticos de enfermagem na for- mação acadêmica. Embora o conhecimento acerca do processo diagnóstico venha sendo divulgado e discutido há pelo menos 40 anos, apenas recentemente foi posto em destaque $o$ pensamento crítico e a necessidade da precisão diagnóstica pelos enfermeiros (8).

Nesse sentido, a imprecisão diagnóstica pode estar atrelada a problemas na graduação, como: desarmonia entre a teoria ministrada em sala de aula e a prática vivenciada nos campos de estágio; ênfase excessiva à escrita do processo de enfermagem em detrimento do entendimento dos alunos sobre os reais problemas de saúde da clientela; e não adoção em campo prático do método diagnóstico de enfermagem em sua rotina (11).

Destarte, são necessárias estratégias de ensino inovadoras, com vistas a auxiliar os estudantes e os profissionais inseridos nos serviços, a refletirem sobre o processo diagnóstico, pensamento crítico e precisão diagnóstica, em virtude da complexidade envolvida nesses conteúdos. Dentre essas estratégias cita-se o uso de estudos de caso simulados e discussões sobre o uso do processo diagnóstico pelos estudantes/profissionais (8).

A literatura assevera que estratégias educativas voltadas para o ensino dos diagnósticos de enfermagem têm sido desenvolvidas com vistas a melhorar o estabelecimento do raciocínio clínico e julgamento diagnóstico, apresentando resultados positivos quanto à melhoria dessas habilidades nos diagnosticadores (12), sendo essas entendidas como um caminho possível para o constante desenvolvimento da capacidade diagnosticadora de estudantes da graduação e profissionais enfermeiros.

Além disso, as universidades de enfermagem do Brasil necessitam de uma reelaboração de seus currículos, as quais devem considerar como eixo norteador dos programas de ensino das disciplinas, do ciclo básico ao profissional, as classificações de enfermagem. Os estudantes devem utilizar-se dos diagnósticos de enfermagem como guia orientador 
dos conteúdos das disciplinas e não mais orientar-se por diagnósticos médicos, de modo que suas habilidades e competências sobre julgamento clínico sejam desenvolvidas a partir das atividades próprias da enfermagem (13).

Ademais, é necessária a implementação da educação continuada nas instituições de saúde e de ensino, com vistas ao treinamento do raciocínio diagnóstico e emprego das taxonomias de enfermagem (10). Assim, além de estratégias para fomentar o processo de raciocínio diagnóstico na graduação, a educação continuada a partir de treinamentos mostra-se eficaz como instrumento para melhorar a acurácia diagnóstica dos enfermeiros inseridos na prática (14). Estudo sobre o assunto ratifica a relevância de treinamentos focando o raciocínio diagnóstico como método efetivo para melhorias da capacidade diagnosticadora de enfermeiros $(2,15)$. Assim, educadores de enfermagem devem criar estratégias educacionais para melhorar as habilidades de raciocínio clínico dos enfermeiros (14).

A literatura revela também que pessoas com um maior grau de titulação apresentam inferência diagnóstica mais precisa. Entretanto, mesmo aqueles com maior titulação, mas que não tiveram a oportunidade de estudar sobre diagnósticos de enfermagem na graduação apresentam maiores dificuldades em estabelecer diagnósticos precisos. Nesse sentido, o ensino sobre as respostas humanas e como interpretá-las deve ser apoiado, sendo uma responsabilidade de professores e gerentes das instituições de saúde (8).

O dado acima citado assemelha-se ao identificado nesta pesquisa, na qual o maior grau de titulação obtido era o mestrado, entretanto, a maioria não apresentava tempo suficiente na prática clínica, podendo ter repercutido nos resultados referentes à precisão diagnóstica da maioria desses. Nesse aspecto, pesquisa apresenta que mesmo com alta titulação acadêmica, ainda assim os enfermeiros apresentavam baixa precisão diag- nóstica (10).

Entretanto, estudo posterior afirma que o grau de acurácia diagnóstica apresentado pelos enfermeiros pode não ter relação com o contato prévio teórico/prático com o processo de enfermagem e diagnósticos de enfermagem (15).

Como limitações essa pesquisa aponta a pequena amostra, entretanto, o tamanho amostral utilizado é o recomendado pela literatura (7).

Conclui-se que menos da metade dos enfermeiros especialistas obtiveram resultado satisfatório referente à inferência diagnóstica. Esse resultado pode ser um reflexo das deficiências do processo de ensino-aprendizagem na temática dos diagnósticos de enfermagem e lacunas na educação continuada.

Dessa forma, torna-se imperativo o desenvolvimento de estratégias que facilitem o aprendizado do discente da graduação e forme enfermeiros com maior capacidade de utilizarem a metodologia própria de sua profissão para o crescimento dessa e do espaço da assistência sistematizada nos campos de trabalho. Outrossim, recomenda-se a realização de educação continuada aos enfermeiros da prática.

A realização deste estudo contribuiu para a identificação das lacunas existentes no processo de inferência diagnóstica desempenhada pelos enfermeiros, apontando a necessidade premente da implantação de novas metodologias de ensino aplicadas na graduação, bem como nos serviços de saúde, tendo-se em vista as repercussões da inferência incorreta para o paciente.

\section{REFERÊNCIAS}

1. Herdman TH, Kamitsuru S, organizadoras. Diagnósticos de enfermagem da NANDA: Definições e classificação 20152017. 10a ed. Machado R, tradução. Porto Alegre: Artmed; 2015. 496 p. 
2. Pereira JMV, Cavalcanti ACD, Lopes MVO, Silva VG, Souza RO, Gonçalves LC. Acurácia na inferência de diagnósticos de enfermagem de pacientes com insuficiência cardíaca. Rev Bras Enferm. 2015; 68(4): 690-96.

3. Carvalho ECC, Cruz DALM, Herdman TH. Contribuição das linguagens padronizadas para a produção do conhecimento, raciocínio clínico e prática clínica da Enfermagem. Rev Bras Enferm. 2013; 66(esp): 134-41.

4. Costa CPV, Luz MHBA. Produção científica da enfermagem sobre raciocínio diagnóstico: revisão integrativa. J Nurs UFPE on line [Internet]. 2016 [citado 12 dic 2016]; 10(1): 152-62. Disponível em: https://periodicos.ufpe.br/revistas/revistaenfermagem/article/view/10933

5. Gimenes FRE, Motta APG, Silva PCS, Gobbo AFF, Atila E, Carvalho EC. Identificação de intervenções de enfermagem associadas à acurácia dos diagnósticos de enfermagem para pacientes com cirrose hepática. Rev Lat Am Enfermagem. 2017; 25(e2933): 1-9.

6. Marini M, Chaves EHB. Evaluation of the accuracy of nursing diagnoses in a Brazilian emergency service. Int J Nurs Terminol Classif. 2011; 22(2): 56-67.

7. Lopes MV, Silva VM, Araujo TL. Methods for establishing the accuracy of clinical indicators in predicting nursing diagnoses. Int J Nurs Knowl. 2012; 23(3): 134139.

8. Lunney M. Pensamento crítico para o alcance de resultados positivos em saúde: análises e estudos de caso em enferma- gem. Porto Alegre: Artmed; 2011. 353 p.

9. Hradesky JL. Productivity \& quality improvement: a practical guide to implementing statistical process control. New York: McGraw-Hill; 1989. 320 p.

10. Morais SCRV, Nóbrega MML, Carvalho EC. Convergências, divergências e acurácia diagnóstica à luz de duas terminologias de enfermagem. Rev Bras Enferm. 2015; 68(6): 1086-92.

11. Santo ADB, Oliveira KKD, Rosário SSD, Lira ALBC, Tourinho FSV, Santos VEP. Strategies for teaching learning process in nursing graduate and postgraduate nursing. J. res.: fundam. care. online [Internet]. 2014 [citado 12 dic 2016]; 6(3): 1212-20. Disponível em: http://www.seer. unirio.br/index.php/cuidadofundamental/article/view/1604

12. Lira ALBC, Lopes MVO. Diagnóstico de enfermagem: estratégia educativa fundamentada na aprendizagem baseada em problemas. Rev Lat Am Enfermagem. 2011; 19(4): 936-943.

13. Cruz ICF. Diagnóstico de Enfermagem: Qual a abordagem no novo currículo? Boletim NEPAE-NESEN [Internet]. 2015 [citado 12 dec 2016]; 12(2): 1-5. Disponível em: http://www.uff.br/

14. Collins A. Effect of continuing nursing education on nurses' attitude toward and accuracy of nursing diagnosis. Int J Nurs Knowl. 2013; 24(3): 122-28.

15. Oliveira IM, Silva RCG. Comparação do grau de acurácia diagnóstica de graduandos e enfermeiros em programas de residência. REME. 2016; 20(e952): 1-8. 